\title{
Gas Expanded Polymer Process to Anneal Nanoparticle Dispersion in Thin Films
}

\author{
Preejith V. Ambuken ${ }^{\dagger+}$, Holly A. Stretz ${ }^{* \dagger}$, Mark Dadmun ${ }^{\| \Delta}$, and S. Michael Kilbey II ${ }^{\| \S}$ \\ ${ }^{\dagger}$ Department of Chemical Engineering, Tennessee Technological University, Cookeville,
} Tennessee 38505, United States

†enter for Manufacturing Research, Tennessee Technological University, Cookeville, Tennessee 38505, United States

"Department of Chemistry, University of Tennessee, Knoxville, Tennessee 37996, United States

${ }^{\Delta}$ Chemical Sciences Division, Oak Ridge National Laboratory, Oak Ridge, Tennessee 37831, United States

${ }^{\S}$ Department of Chemical and Bimolecular Engineering, University of Tennessee, Knoxville, TN 37996, United States

Corresponding author: Holly A. Stretz, hstretz@tntech.edu, 931-372-3495

\begin{abstract}
Organic photovoltaic (OPV) active layers made by spin-coating a solution comprising poly(3hexylthiophene) (P3HT) and phenyl-C61-butyric acid methyl ester (PCBM) nanoparticles have been shown to adopt a non-uniform concentration profile across the thin film dimension. This inhomogeneous distribution can reduce the efficiency of the device. In the present work a new process, gas expanded polymer (GXP) annealing, is applied to P3HT/PCBM thin film blends,
\end{abstract}


enabling the distribution of the PCBM nanoparticles to be manipulated by varying the GXP processing conditions. Films of $50 \mathrm{~nm}$ thickness (nominally) created by spin casting a blend of P3HT mixed with PCBM were annealed by oscillatory GXP and GXP at constant pressure using high pressure $\mathrm{CO}_{2}$. An increase in P3HT crystallinity (detected by X-ray diffraction and UV-Vis spectroscopy) along with a more uniform distribution of PCBM nanoparticles in the thickness dimension, as interpreted from neutron reflectivity measurements, were observed after oscillatory GXP annealing. In addition, static water contact angles suggest that the film/air interface is enriched/depleted in PCBM relative to the as-cast film. These results demonstrate that GXP annealing, which is commercially scalable, can be successfully used to create a uniform distribution of PCBM nanoparticles across the thickness dimension in a P3HT thin film.

\section{Introduction}

Organic photovoltaic (OPV) devices convert solar energy into electrical energy. The potential advantages of OPVs over silicon-based devices include ease of processing and their light weight. Among the different active layer materials that have been investigated, blends of poly(3hexylthiophene) (P3HT) and phenyl-C61-butyric acid methyl ester (PCBM) fullerenes are often used as a model system. OPVs based on the bulk heterojunction (BHJ) blend concept also exhibit modest power conversion efficiencies, reaching up to $10.6 \%$ for tandem arrangement.[1] Because the exciton generated upon photon adsorption has a very short lifetime, it is important to create BHJ domain sizes that match the short exciton diffusion length.[2] In terms of structure and performance, the "rivers and streams" structural model for nanoparticle dispersion proposed by Yin et al.[3] describes a theoretical optimal hierarchy. Yin et al. determined through small angle neutron scattering that three phases exist in the P3HT/PCBM system: a pure crystalline 
P3HT phase, a PCBM-rich phase and an amorphous miscible phase of P3HT and PCBM.[3] According to this model, the amorphous P3HT/PCBM domain consists of a large number of donor/acceptor interfaces where the tightly bound excitons can dissociate. These charges then flow to the less dispersed "river" phases to transport to their respective electrode.[3] Along the axis from one electrode to the other, the depth profile of the PCBM should not preferentially collect at the anode,[4] as a higher concentration of PCBM at the anode results in lower device efficiency.[5, 6] Thus a mixed morphology along all axes combined with interconnected domains is desirable. The current spin coating process when used alone makes this complex structure difficult to control in a scalable, cost effective manner.

Prior work indicates that device efficiency and dispersion of nanoparticles can be improved in spin-cast nanocomposite films if they are subsequently exposed to thermal annealing $[5,7,8]$ or solvent annealing.[4, 9-11] Dang et al. have given a more exhaustive review of how thermal annealing and solvent annealing are known to affect both P3HT and PCBM morphology and the intimate dependence on P3HT regioregularity and molecular weight considerations.[12] In thermal annealing, heat treatment leads to improved P3HT crystallinity, which leads to an improvement in charge transport from grain to grain. $[13,14]$ Thermal annealing also introduces the possibility of macrophase separation, which can reduce device performance.[6, 15] Thermal annealing requires high heating and cooling rates for uniform heat transfer throughout the sample. Studies show that solvent vapor annealing improves the crystallinity of the polymer as well. $[6,16]$ Solvent vapor annealing; however, is often not environmentally friendly due to the nature of organic solvents commonly used in connection with solvating P3HT. Thus, while both thermal and solvent annealing offer the advantage of improving P3HT crystallinity, the 
concomitant effect of coarsening the dispersion by occlusion of PCBM, often to the extent that nanoparticle aggregates occupy micron-scale domains, is deleterious.

Kiel et al. used neutron reflectivity to show that thermal annealing of P3HT/PCBM bulk heterojunctions at $170{ }^{\circ} \mathrm{C}$ led to the PCBM concentration being highest at the polymer/substrate interface both before and after annealing[17]. Germack et al. used near-edge X-ray absorption fine structure spectroscopy to arrive at similar results.[18] Chen et al., also using neutron reflectivity measurements, showed that PCBM selectively aggregates at the polymer/air interface when P3HT/PCBM bilayers are thermally annealed.[5] Parnell et al. also used neutron reflectivity to show that $\mathrm{P} 3 \mathrm{HT} / \mathrm{PCBM}$ films, when subjected to either solvent vapor or thermal annealing, showed an increase in concentration of PCBM at the polymer/air interface.[4] Thus, while either thermal or solvent vapor annealing are viable processes for changing the distribution of PCBM nanoparticles in a polymer film, neither method offers a process to limit the enhancement of PCBM at the anode where the holes are extracted.

In this work, gas expanded polymer (GXP) annealing is examined for the first time as an alternate method to control the distribution of PCBM nanoparticles in BHJ films. In particular, carbon dioxide $\left(\mathrm{CO}_{2}\right)$ is used as a high pressure gas or supercritical fluid to create a gas expanded $\mathrm{P} 3 \mathrm{HT} / \mathrm{PCBM}$ film. $\mathrm{CO}_{2}$ is advantageous because it is a nontoxic and inexpensive fluid, and GXP processing using $\mathrm{CO}_{2}$ is scalable; it is used in polymer synthesis and processing, [1921] where processes share a number of similarities to roll-to-roll printing and coating processes. [22] In addition, the solvating properties of the fluid can be quickly and easily controlled by changes in temperature and pressure. While there have been numerous works over the past two decades showing the effect of $\mathrm{CO}_{2}$ swelling of liquids (termed gas expanded liquids or GXL) 
resulting in novel distributions of nanoparticles on surfaces[23], only a handful of works have shown the possibility of using $\mathrm{CO}_{2}$ swelling of polymers to process polymer nanocomposites. For dodecanethiol-capped $\mathrm{Ag}$ and $\mathrm{CdSe} / \mathrm{ZnS}$ nanoparticles dispersed in hexane, Anand et al. showed that it is possible to precipitate nanoparticles in a size selective fashion by controlling $\mathrm{CO}_{2}$ vapor pressure.[24-26] A balance of Van der Walls and steric interactions, as well as the physicochemical properties of the particles and of the solvent, were shown to mediate the deposition, which could be explained using Flory Huggins model and DLVO theory. Bhosale et al. have demonstrated that deposition and gas expanded liquid annealing produce a uniform monolayer of gold nanoparticles over wide areas when dispersed in $\mathrm{CO}_{2}$-expanded hexane.[27] A critical range of $\mathrm{CO}_{2}$ pressure was necessary to control the organization of the gold nanoparticle monolayer film. By oscillating $\mathrm{CO}_{2}$ around a critical pressure, positional defects in the monolayer of decorated nanoparticles could be removed. While Bhosale's work demonstrated that oscillation of solvation power can anneal nanoparticle-containing thin films, by extension it holds promise for affecting PCBM dispersion in a P3HT/PCBM thin film. In fact, Amonoo et al.[28] used supercritical $\mathrm{CO}_{2}$ to anneal a P3HT/PCBM thin film. While the processing did not include pressure oscillation or detailed morphological characterization through the thin dimension of the film, from energy filtered electron microscopy imaging and UV-Vis spectroscopy they found that morphological changes did occur and that the power conversion efficiency (PCE) of an OPV device could be improved beyond the gain imparted by thermal annealing: Specifically, their devices displayed a PCE of $2.9 \%$ when annealed in supercritical $\mathrm{CO}_{2}$ at 1500 psi and $50^{\circ} \mathrm{C}$ versus $2.6 \%$ PCE for their thermally annealed system. The present paper reports more uniform dispersion of PCBM nanoparticles in a P3HT thin film detected through the thickness of the film. $\mathrm{CO}_{2}$ gas expanded polymer anneal processing was 
used to effect the changes noted, and the solvating power was varied through pressure oscillations during processing versus constant pressure. The uniform concentration profile of PCBM achieved in this process has not been previously reported.

2. Materials and Methods

\subsection{Materials}

[6,6]-Phenyl-C61-butyric acid methyl ester (PCBM, $>99.5 \%$ ) and 91-94\% regioregular poly (3-hexylthiophene) (P3HT), were purchased from Nano-C, Inc. and Rieke Metals, Inc., respectively, and used as received. Silicon wafers were used as substrates for neutron reflectivity measurements (3" diameter, $\mathrm{P}(100)$ University Wafer, donated by Hemlock Semiconductor) and glass substrates were used for X-ray diffraction, ultraviolet visible (UV/vis) spectroscopy and contact angle measurements. Chlorobenzene $(99 \%+$, Acros Organics) was used as received and high purity grade $\mathrm{CO}_{2}(99.9 \%$, Airgas, Inc) was used.

\subsection{Sample Preparation}

To fabricate thin films, P3HT and PCBM were co-dissolved in chlorobenzene (CB) at a total solids concentration of $10 \mathrm{mg} / \mathrm{ml}$ with $\mathrm{P} 3 \mathrm{HT}$ and $\mathrm{PCBM}$ mixed in a mass ratio of $1: 1$. For neutron reflectivity studies, silicon wafers were cleaned by immersion in Piranha solution (a 3:1 $(\mathrm{v} / \mathrm{v})$ mixture of concentrated sulfuric acid and $30 \%(\mathrm{v} / \mathrm{v})$ hydrogen peroxide) which was heated to $80^{\circ} \mathrm{C}$. Caution: Piranha solution should be handled with care: as it is a strong acid and oxidizer, reacting violently with organic compounds. After immersion for $30 \mathrm{~min}$, the wafers 
were removed, rinsed with high purity water, and dried under dry nitrogen. The freshly cleaned wafer was then exposed to UV light (185-254 nm) for 5 minutes. All films were made by spin coating the P3HT/PCBM solution at $400 \mathrm{rpm}$ for 4 minutes and then at $1000 \mathrm{rpm}$ for 20 seconds. For X-ray diffraction, UV-Vis and contact angle measurements, the P3HT/PCBM thin films were created by an identical spin coating procedure on clean glass slides. All glass slides were cleaned by sonicating the substrate sequentially for 15 minutes in soapy water, distilled water, acetone and then isopropanol. After sonication, the glass slides were dried with a stream of nitrogen gas. Material designations used in this paper for all the samples are shown in Table 1.

\section{[Insert Table 1]}

\subsection{GXP Annealing}

A 2 L Anton Paar pressure vessel was installed in an Applied Separation Spe-ed ${ }^{\mathrm{TM}}$ oven with a temperature controller. This pressure vessel was coupled with two ISCO 500D syringe pumps for continuous $\mathrm{CO}_{2}$ delivery. Two different protocols - oscillating and constant pressure exposures were used to anneal P3HT/PCBM thin films. In each case, after sealing the sample in the vessel, it was flushed with $\mathrm{CO}_{2}$ for 40 minutes in order to completely purge air from the system.

As shown in Figure 1, for the oscillation-annealed process, the pressure was first increased to a base pressure (e.g. $675 \mathrm{psi}$ ) by pumping $\mathrm{CO}_{2}$ at a flow rate of $100 \mathrm{ml} / \mathrm{min}$. Once this pressure was reached, oscillatory cycles were commenced by slowly pressurizing the vessel to $4.83 \mathrm{MPa}$ (700 psi) by pumping $\mathrm{CO}_{2}$ at a flow rate of $3 \mathrm{ml} / \mathrm{min}$, followed by depressurizing to $4.65 \mathrm{MPa}$ (675 psi) using the same low flow rate of $3 \mathrm{ml} / \mathrm{min}$. A slow flow rate was used because Bhosale et al. suggested that slower flow rates led to more uniform films.[27] 
The pressure oscillation was repeated three times, and then the vessel was depressurized by opening the vent valve. We identify this process as 700/OSC (maximum pressure/oscillatory cycling) and note that per Table 1 we also tested a higher maximum pressure, 900/OSC.

A second type of GXP annealing process based on holding the pressure constant (no oscillation) was also examined. In this process, the vessel was pressurized with $\mathrm{CO}_{2}$ at a flow rate of $100 \mathrm{ml} / \mathrm{min}$ to either $700 \mathrm{psi}$ or to $900 \mathrm{psi}$. This pressure was held for 30 minutes, after which time the vessel was depressurized by opening the vent valve. We identify these processes as $700 / 30$ and $900 / 30$, in reference to the hold pressure and hold time. In all cases, after venting the vessel, the film-coated substrates were removed and quickly transferred to and stored in a vacuum desiccator until further characterization.

\subsection{X-ray Diffraction}

X-ray diffraction (XRD) measurements were made using a Rigaku Ultima IV X-ray Diffractometer. All samples were tested using parallel beam mode from $2^{\circ}-8^{\circ}$ with a scintillation counter and a monochromator. The source angle was fixed at $0.3^{\circ}$ for all of the scans. Repeat measurements were made using different as-cast and annealed samples to verify reproducibility of results.

\subsection{Ultraviolet-visible Spectroscopy}

The absorption spectra of pristine P3HT/PCBM films and pressure annealed P3HT/PCBM films on glass were obtained using a Varian Cary 50 Ultraviolet-Visible Spectrophotometer. Baseline corrections were performed using a clean glass substrate. 


\subsection{Contact Angle}

Static water contact angles were determined from the shape of a sessile drop created by dropping $2 \mu \mathrm{l}$ of distilled water on the pressure annealed and pristine P3HT/PCBM thin films made on glass slides. An average value was determined from six measurements taken at different locations on the film. The shape of the water droplet was digitally captured and analyzed using an open source image processing program, Image $J$, to determine the contact angle.

\subsection{Neutron Reflectivity}

All reflectivity measurements were completed on the Liquids Reflectometer at the Spallation Neutron Source at Oak Ridge National Laboratory using a continuous wavelength band $(2.75<\lambda$ $<5.75 \AA$ A). Specular reflectivity was collected at several different angles, $\theta$, equating to a wave vector transfer $(Q=(4 \pi / \lambda) \sin \theta)$ range of $0.006<Q<0.16 \AA$. Incident beam slits were set to maintain a constant wave vector resolution $\delta Q / Q=0.02$, which allows the data at different angles to be "stitched" together into a single reflectivity curve.[29] The measured reflectivity was modeled using a series of "slab-like" layers described by their neutron scattering length density, which depends on composition, thickness and roughness (calculated as the full-width at half maximum). The model was constrained by a balance of the scattering length densities across all layers and optimized for goodness-of-fit which is represented by $\chi^{2}$ :

$$
\chi^{2}=\frac{\sum\left(R_{(q) \text { calc }}-R_{(q) \exp }\right)^{2}}{R_{q(\exp )}^{2}}
$$


where $R_{(\mathrm{q}) \text { calc }}$ and $R_{(\mathrm{q}) \exp }$ are the calculated and experimental reflectivity, respectively. The PCBM concentration depth profile, $\phi(\mathrm{z})$, is found using equation 2:

$$
\varphi(z)_{P C B M}=\frac{\left(\rho_{(Z)}-\rho_{(P 3 H T)}\right)}{\left(\rho_{(P C B M)}-\rho_{(P 3 H T)}\right)}
$$

Here $\varphi(\mathrm{z}), \rho_{(\mathrm{z})}, \rho_{(\mathrm{P} 3 \mathrm{HT})}$ and $\rho_{(\mathrm{PCBM})}$ are the volume fraction of PCBM at depth $z$, experimental scattering length density at depth $z$, scattering length density of P3HT and scattering length density of PCBM, respectively. Scattering length densities were calculated based on the atomic composition and density.[30] The scattering length densities of amorphous and crystalline P3HT were taken as $0.62 \times 10^{-6} \AA^{-2}$ and $0.69 \times 10^{-6} \AA^{-2}$ respectively. The density of crystalline P3HT was taken as $1.12 \mathrm{~g} \mathrm{~cm}^{-3} \cdot[5,31]$ The density of amorphous P3HT was estimated as $10 \%$ less than that of crystalline P3HT.[5] The scattering length density of amorphous PCBM is calculated as $4.4 \times 10^{-6} \AA^{-2}$. (Here again it was assumed that amorphous PCBM is $10 \%$ less dense than crystalline PCBM.) The density of crystalline PCBM was reported as $1.67 \mathrm{~g} / \mathrm{cm}^{-3}$ by Rispen et al.[5, 32] The silicon wafers were 3 inches diameter and 0.015 inch thick.

\section{Results and Discussion:}

Previous studies have indicated that PCBM fullerenes preferentially enrich the silicon substrate when 1/1 P3HT/PCBM solutions are spin cast, and this enhancement in PCBM persists when the films (on Si) are thermally annealed.[17] The theories for the driving force for PCBM migration range from enhancement of the amorphous P3HT layer at surfaces (where PCBM is miscible only in the amorphous layer - see Keum et al. [33]) versus interfacial tension as a driving force 
for migration (see Pavlopoulou et al. [34]). Pavlopoulou et al. argue that depending on whether the device is an inverted solar cell $\left(\mathrm{TiO}_{\mathrm{x}}\right)$ or a conventional OPV architecture (PEDOT:PSS layer), this stratification of PCBM could either be favorable or detrimental (respectively) to the power conversion efficiency of the device. Guaralnick et al. claim that annealing had little effect on the vertical PCBM concentration profile when PEDOT:PSS was at the interface with the bulk heterojunctions and that all studies relevant to bulk heterojunctions should include the presence of a buffer layer. The profiles obtained by Keum et al. in their studies show much more significant changes. One difference is the 1.0/0.7 mass ratio of P3HT/PCBM was used by Keum et al., while the other two studies used a 1.0/1.0 ratio for the BHJ blend. As the miscibility limit for PCBM in P3HT is close to $20 \%$ (v/v) [5] one might argue that the oversaturation of PCBM could also have a very large effect in stratification and simultaneously impact the extent of crystallization. Given these various considerations and that the present work is focused on understanding the effects of GXP annealing on stratification within the active layer, no buffer layer was incorporated into the present studies. A GXP annealing process was implemented in an attempt to achieve a more uniform distribution of PCBM in the thickness dimension. We monitor the film morphology using X-ray diffraction and UV-Visible spectroscopy to characterize polymer ordering and crystallization. Contact angle measurements provide information about the relative amount of the two components at the film/air interface. Neutron reflectivity studies, due to the neutron scattering contrast between P3HT and PCBM, provide insight into the laterally-averaged composition depth profile.

\subsection{X-ray Diffraction:}


X-ray diffraction analyses for all the samples were recorded and two representative curves are shown in Figure 2. The degree of P3HT crystallinity for the pristine, "as-cast" P3HT/PCBM was quite low. After GXP annealing, all of the samples exhibit a peak at $2 \theta=5.20^{\circ}$ which is characteristic of the $<100>$ peak of P3HT crystals. This development is attributed to an increase in P3HT crystallinity as a consequence of the annealing process. Scans obtained from the samples that experienced the 700/30, 900/OSC and 900/30 GXP process also exhibited an enhancement in the $<100>$ peak. In all of the samples, the peak intensity varied by less than $+5 \%$ and the peak position remained the same. All samples were cast in the same fashion and thus the masses of the films were approximately the same, as observed from the film thicknesses reported in Table 1.

\subsection{Ultraviolet Visible Spectroscopy:}

Figure 3 shows the effect of GXP annealing on the UV-Vis absorption spectra of the P3HT/PCBM thin films. For the pristine, as-cast P3HT films the $\lambda_{\max }$ is observed at $501 \mathrm{~nm}$ with small shoulders at $555 \mathrm{~nm}$ and $595 \mathrm{~nm}$. After GXP annealing all samples exhibited a red shift in $\lambda_{\max }$ to $515 \mathrm{~nm}$ (splitting at $545 \mathrm{~nm}$ ) and the vibronic shoulder at $595 \mathrm{~nm}$ became more distinguished.[35, 36] This red shift signifies stronger interchain interactions and ordering of the P3HT chains. $[35,36]$ These results signify that $\mathrm{CO}_{2}$ penetrated into the bulk heterojunction thin film and facilitated the diffusive transport of P3HT chains, which led to the observed enhancement in P3HT ordering.[11]

\subsection{Contact Angle Measurements:}

Contact angle measurements were performed to monitor the movement of PCBM to or from the polymer/air interface. The contact angles for all of the films, which were made by spin 
coating on glass, are reported in Table 1. While the ability of contact angle measurements to discriminate differences between P3HT and PCBM is limited by the small difference in contact angle between $\mathrm{P} 3 \mathrm{HT}$ and $\mathrm{PCBM}$ (approximately $8^{\circ}$ ), the results show that all of the pressure annealed samples exhibit a lower contact angle than that of the as-cast film. This change in contact angle is therefore consistent with a redistribution of PCBM nanoparticles during GXP annealing from the silicon/film interface toward the air/film interface. The as-cast P3HT/PCBM film had a contact angle that was closer to that of pure P3HT, which is consistent with an excess of P3HT chains at the polymer/air interface. Because changes in surface roughness could also account for changes in contact angle, PCBM concentration profiles were examined more rigorously using neutron reflectivity.

\subsection{Neutron Reflectivity:}

The specular neutron reflectivity (NR) curves as well as the model fits of these curves are presented in Figure 4. The data are plotted as $R Q^{4}$ versus $Q$ to compensate for the Fresnel decay above the critical edge $\left(Q_{\mathrm{c}}\right)$, which also highlights the features of the data more clearly. Figure $4 \mathrm{a}$ is the reflectivity measured for a pristine, as-cast P3HT/PCBM film while Figures $4 b$, 4c and 4d show the reflectivity curve for each of the three pressure annealed thin films.

The SLD profile, $\rho(z)$, of the active layer thin film was determined by modeling the measured reflectivity to extract the concentration (volume percent) profile of PCBM in thin films, $\phi(z)$, according to Equation 2. Because of sample-to-sample variation in film thickness and in order to facilitate direct comparison, the concentration profiles of PCBM determined from this analysis as a function of normalized thickness are shown in Figure 5. The concentration profile in the dimension of the film thickness for the as-cast sample indicates an excess of PCBM at the silicon 
substrate with the PCBM concentration decreasing with increasing distance from the silicon substrate. This result is similar to what others note for as-cast samples, including Parnell et al. and Hegde et al.[4, 37]

The samples subjected to GXP annealing show different behaviors: In the 700/OSC sample, the excess of PCBM observed at the film/substrate interface in the as-cast film appears to shift toward the polymer/air interface, although in both cases PCBM remains enriched in the region close to the silicon substrate. On the other hand, the PCBM concentration profile determined for the films subjected to GXP annealing at 700/30 and 900/OSC is nearly uniform across the film thickness dimension. The 900/OSC film has the lowest concentration of PCBM at the film/silicon interface, which indicates that oscillatory GXP annealing with $\mathrm{CO}_{2}$ at the higher pressure homogenizes the distribution of the nanoparticles through the center of the polymer film. After GXP annealing, there is reduced concentration of PCBM at the silicon/polymer interface. The most direct comparison to literature reports is to the neutron reflectivity work by Parnell et al., where both thermal and solvent annealing altered the PCBM depth profile through diffusion of PCBM away from the Si surface, but neither process resulted in as significant of transport of the PCBM away from the Si surface as we see in oscillated GXP.[4]

As shown in Table 1, swelling of the bulk heterojunction thin films was observed after GXP annealing. An increase of approximately $110 \AA-120 \AA$ (nominally 20\%) was observed compared to the as-cast P3HT/PCBM film. Asada et al.[38] and Koga et al.[39] also observed that the thickness of polystyrene and poly(methyl methacrylate) films increased after exposing them to supercritical $\mathrm{CO}_{2}$, and this effect was attributed to the expansion of polymer chains by excess absorption of $\mathrm{CO}_{2}$ molecules.[38] Asada et al. also observed the disappearance of surface 
segregation of nanostructures in polystyrene/PCBM thin films for film thicknesses less than 450 $\AA$ with exposure to supercritical $\mathrm{CO}_{2} \cdot[38]$ Thus these results are consistent with a mechanism such that when P3HT/PCBM thin films are exposed to high pressure $\mathrm{CO}_{2}$, the swelling of P3HT chains aides in the diffusion of $\mathrm{CO}_{2}$-wetted nanoparticles within the films. In total, these results clearly demonstrate that the novel annealing process, GXP, can be used to produce a more homogenous distribution as a function of depth in a thin film during polymer nanocomposite film device processing. Moreover, GXP is a scalable, environmentally-friendly process that is compatible with existing manufacturing infrastructure, such as roll-to-roll processing.

\section{Conclusions}

The modification of the depth profile of P3HT/PCBM thin films using a novel gas expanded polymer (GXP) annealing or high pressure GXP annealing process is reported. The results indicate that exposure of the nanocomposite thin films to $\mathrm{CO}_{2}$ effectively altered the crystallinity and the concentration profile of PCBM in the thickness dimension, presumably by inducing mobility of the components as $\mathrm{CO}_{2}$ plasticized the polymer. Neutron reflectivity, which is sensitive to the distribution of nanoparticles in the P3HT/PCBM thin films, reveals significant changes in the morphology as a function of $\mathrm{CO}_{2}$ pressure and annealing protocol. The as-cast P3HT/PCBM thin films exhibit a slight excess of PCBM segregated near the silicon substrate. Exposure to $\mathrm{CO}_{2}$ facilitates the crystallization of $\mathrm{P} 3 \mathrm{HT}$, as well as expansion of amorphous polymer chains, which helps to accommodate nanoparticle movement towards a more homogenous distribution of nanoparticles throughout the depth of the film. The P3HT/PCBM thin film that was exposed to GXP annealing with $\mathrm{CO}_{2}$ (with the pressure oscillated between 875 and 900 psi (900/OSC)) results in a fairly homogeneous dispersion of fullerenes throughout the 
depth of the film. Therefore, the gas expanded annealing procedure provides a means of controlling the distribution of nanoparticles in the film without the use of toxic solvents.

Acknowledgements

Neutron reflectivity measurements were conducted at the Spallation Neutron Source, which is sponsored at Oak Ridge National Laboratory by the Scientific User Facilities Division, Office of Basic Energy Sciences, US Department of Energy. The authors gratefully acknowledge the support of this work by the NSF-funded TN-SCORE program (NSF EPS-1004083). The authors also thank Ryan Roman, Brian Daniels, Hardik Patel, Alex Holburn, Renaldo Erskine, Luke Mirtes, Dr. Huipeng Chen, Adam Imel and Zach Seibers for helpful discussions and early investigations that set the stage for this work. Hemlock Semiconductor is gratefully acknowledged for the donation of silicon wafers used in this research. Some funding for students was provided by the Tennessee Technological University Center for Manufacturing Research.

References

[1] J. You, L. Dou, K. Yoshimura, T. Kato, K. Ohya, T. Moriarty, K. Emery, C.-C. Chen, J. Gao, G. Li, Y. Yang, A polymer tandem cell with $10.6 \%$ power conversion efficiency, Nature Communications, 4 (2013) 1446.

[2] H. Hoppe, N.S. Sariciftci, Organic solar cells: An overview, Journal of Materials Research, 19 (2004) 1924-1945.

[3] W. Yin, M. Dadmun, A new model for the morphology of P3HT/PCBM organic photovoltaics from small-angle neutron scattering: rivers and streams, ACS Nano, 5 (2011) 4756-4768. 
[4] A.J. Parnell, A.D. Dunbar, A.J. Pearson, P.A. Staniec, A.J. Dennison, H. Hamamatsu, M.W. Skoda, D.G. Lidzey, R. Jones, Depletion of PCBM at the cathode interface in P3HT/PCBM thin films as quantified via neutron reflectivity measurements, Advanced materials, 22 (2010) 24442447.

[5] H. Chen, R. Hegde, J. Browning, M. Dadmun, The miscibility and depth profile of PCBM in P3HT: thermodynamic information to improve organic photovoltaics, Physical Chemistry Chemical Physics, 14 (2012) 5635-5641.

[6] R. Hegde, N. Henry, B. Whittle, H. Zang, B. Hu, J. Chen, K. Xiao, M. Dadmun, The impact of controlled solvent exposure on the morphology, structure and function of bulk heterojunction solar cells, Solar Energy Materials and Solar Cells, 107 (2012) 112-124.

[7] T.M. Clarke, A.M. Ballantyne, J. Nelson, D.D. Bradley, J.R. Durrant, Free energy control of charge photogeneration in polythiophene/fullerene solar cells: the influence of thermal annealing on P3HT/PCBM blends, Advanced Functional Materials, 18 (2008) 4029-4035.

[8] L. Zeng, C.W. Tang, S.H. Chen, Effects of active layer thickness and thermal annealing on polythiophene: Fullerene bulk heterojunction photovoltaic devices, Applied Physics Letters, 97 (2010) 053305.

[9] S. Miller, G. Fanchini, Y.-Y. Lin, C. Li, C.-W. Chen, W.-F. Su, M. Chhowalla, Investigation of nanoscale morphological changes in organic photovoltaics during solvent vapor annealing, Journal of materials chemistry, 18 (2008) 306-312.

[10] H. Tang, G. Lu, L. Li, J. Li, Y. Wang, X. Yang, Precise construction of PCBM aggregates for polymer solar cells via multi-step controlled solvent vapor annealing, Journal of Materials Chemistry, 20 (2010) 683-688.

[11] Y. Zhao, Z. Xie, Y. Qu, Y. Geng, L. Wang, Solvent-vapor treatment induced performance enhancement of poly (3-hexylthiophene): methanofullerene bulk-heterojunction photovoltaic cells, Applied physics letters, 90 (2007) 043504-043504-043503.

[12] M.T. Dang, L. Hirsch, G. Wantz, J.D. Wuest, Controlling the morphology and performance of bulk heterojunctions in solar cells. Lessons learned from the benchmark poly(3hexylthiophene):[6,6]=phenyl-C61-butyric acid metyl ester sytem, Chemical Reviews, 113 (2013) 3734-3765.

[13] W. Ma, C. Yang, X. Gong, K. Lee, A.J. Heeger, Thermally stable, efficient polymer solar cells with nanoscale control of the interpenetrating network morphology, Advanced Functional Materials, 15 (2005) 1617-1622.

[14] I. Riedel, V. Dyakonov, Influence of electronic transport properties of polymer-fullerene blends on the performance of bulk heterojunction photovoltaic devices, Physica Status Solidi (A), 201 (2004) 1332-1341. 
[15] S. Bertho, G. Janssen, T.J. Cleij, B. Conings, W. Moons, A. Gadisa, J. D’Haen, E. Goovaerts, L. Lutsen, J. Manca, Effect of temperature on the morphological and photovoltaic stability of bulk heterojunction polymer: fullerene solar cells, Solar Energy Materials and Solar Cells, 92 (2008) 753-760.

[16] J.H. Park, J.S. Kim, J.H. Lee, W.H. Lee, K. Cho, Effect of annealing solvent solubility on the performance of poly (3-hexylthiophene)/methanofullerene solar cells, The Journal of Physical Chemistry C, 113 (2009) 17579-17584.

[17] J.W. Kiel, B.J. Kirby, C.F. Majkrzak, B.B. Maranville, M.E. Mackay, Nanoparticle concentration profile in polymer-based solar cells, Soft Matter, 6 (2010) 641-646.

[18] D.S. Germack, C.K. Chan, B.H. Hamadani, L.J. Richter, D.A. Fischer, D.J. Gundlach, D.M. DeLongchamp, Substrate-dependent interface composition and charge transport in films for organic photovoltaics, Applied Physics Letters, 94 (2009) 233303.

[19] D.L. Tomasko, H. Li, D. Liu, S. Han, M.J. Wingert, L.J. Lee, K.W. Koelling, A review of $\mathrm{CO} 2$ applications in the processing of polymers, Industrial \& Engineering Chemistry Research, 42 (2003) 6431-6456.

[20] R.B. Yoganathan, R. Mammucari, N.R. Foster, Dense gas processing of polymers, Polymer Reviews, 50 (2010) 144-177.

[21] M. Bhamidipati, A.M. Curto, M.S. Detamore, The future of carbon dioxide for polymer processing in tissue egineering, Tissue Engineering: Part B, 19 (2013) 221-232.

[22] R.R. Sondergaard, M. Hosel, F.C. Krebs, Roll-to-roll fabrication of large are functional organic materials, Journal of Polymer Science B, (2013).

[23] K.P. Johnston, S.R.P. da Rocha, Colloids in supercritical fluids over the last 20 years and future directions, Journal of Supercritical Fluids, 47 (2009) 523-530.

[24] M. Anand, M.C. McLeod, P.W. Bell, C.B. Roberts, Tunable solvation effects on the sizeselective fractionation of metal nanoparticles in $\mathrm{CO} 2$ gas-expanded solvents, The Journal of Physical Chemistry B, 109 (2005) 22852-22859.

[25] M. Anand, L.A. Odom, C.B. Roberts, Finely controlled size-selective precipitation and separation of $\mathrm{CdSe} / \mathrm{ZnS}$ semiconductor nanocrystals using CO2-gas-expanded liquids, Langmuir, 23 (2007) 7338-7343.

[26] M. Anand, S.-S. You, K.M. Hurst, S.R. Saunders, C.L. Kitchens, W.R. Ashurst, C.B. Roberts, Thermodynamic analysis of nanoparticle size selective fractionation using gasexpanded liquids, Industrial \& Engineering Chemistry Research, 47 (2008) 553-559.

[27] P.S. Bhosale, H.A. Stretz, Gold Nanoparticle Deposition Using CO2 Expanded Liquids: Effect of Pressure Oscillation and Surface- Particle Interactions, Langmuir, 24 (2008) 12241 12246. 
[28] J.A. Amonoo, E. Glynos, X.C. Chen, P.F. Green, An Alternative Processing Strategy for Organic Photovoltaic Devices Using a Supercritical Fluid, The Journal of Physical Chemistry C, 116 (2012) 20708-20716.

[29] C. Deodhar, E. Soto-Cantu, D. Uhrig, P. Bonnesen, B.S. Lokitz, J.F. Ankner, S.M. Kilbey, Hydration in Weak Polyelectrolyte Brushes, ACS Macro Letters, 2 (2013) 398-402.

[30] www.ncnr.nist.gov/resources/activation, in.

[31] T. Prosa, M. Winokur, J. Moulton, P. Smith, A. Heeger, X-ray structural studies of poly (3alkylthiophenes): an example of an inverse comb, Macromolecules, 25 (1992) 4364-4372.

[32] M.T. Rispens, A. Meetsma, R. Rittberger, C.J. Brabec, N.S. Sariciftci, J.C. Hummelen, Influence of the solvent on the crystal structure of PCBM and the efficiency of MDMO-PPV: PCBM 'plastic'solar cells, Chemical Communications, (2003) 2116-2118.

[33] J.K. Keum, J.f. Browning, K. Xiao, M. Shao, C. Halbert, K. Hong, Morphological origin for the stratification of P3HT:PCBM blend film studied by neutron relectrometry, Appliec Physics Letters, 103 (2013) 223301.

[34] E. Palopoulou, G. Fleury, D. Deribew, F. Cousin, M. Goeghegan, G. Hadziioannou, Phase separatio-driven stratification in conventional and inverted P3HT:PCBM organic solar cells, Organic Electronics, 14 (2013) 1249-1254.

[35] G. Li, V. Shrotriya, J. Huang, Y. Yao, T. Moriarty, K. Emery, Y. Yang, High-efficiency solution processable polymer photovoltaic cells by self-organization of polymer blends, Nature materials, 4 (2005) 864-868.

[36] G. Li, V. Shrotriya, Y. Yao, Y. Yang, Investigation of annealing effects and film thickness dependence of polymer solar cells based on poly (3-hexylthiophene), Journal of Applied Physics, 98 (2005) 043704-043704-043705.

[37] R. Hedge, N. Henry, B. Whittle, H. Zang, B. Hu, J. Chen, K. Xiao, M. Dadmun, The impact of conrolled solvent exposure on the morphology, structure and function of bulk heterojunction solar cells, Solar Energy and Materials, 107 (2012) 112-124.

[38] M. Asada, P. Gin, M.K. Endoh, S.K. Satija, T. Taniguchi, T. Koga, Directed self-assembly of nanoparticles at the polymer surface by highly compressible supercritical carbon dioxide, Soft Matter, 7 (2011) 9231-9238.

[39] T. Koga, Y.-S. Seo, K. Shin, Y. Zhang, M. Rafailovich, J. Sokolov, B. Chu, S. Satija, The role of elasticity in the anomalous swelling of polymer thin films in density fluctuating supercritical fluids, Macromolecules, 36 (2003) 5236-5243. 


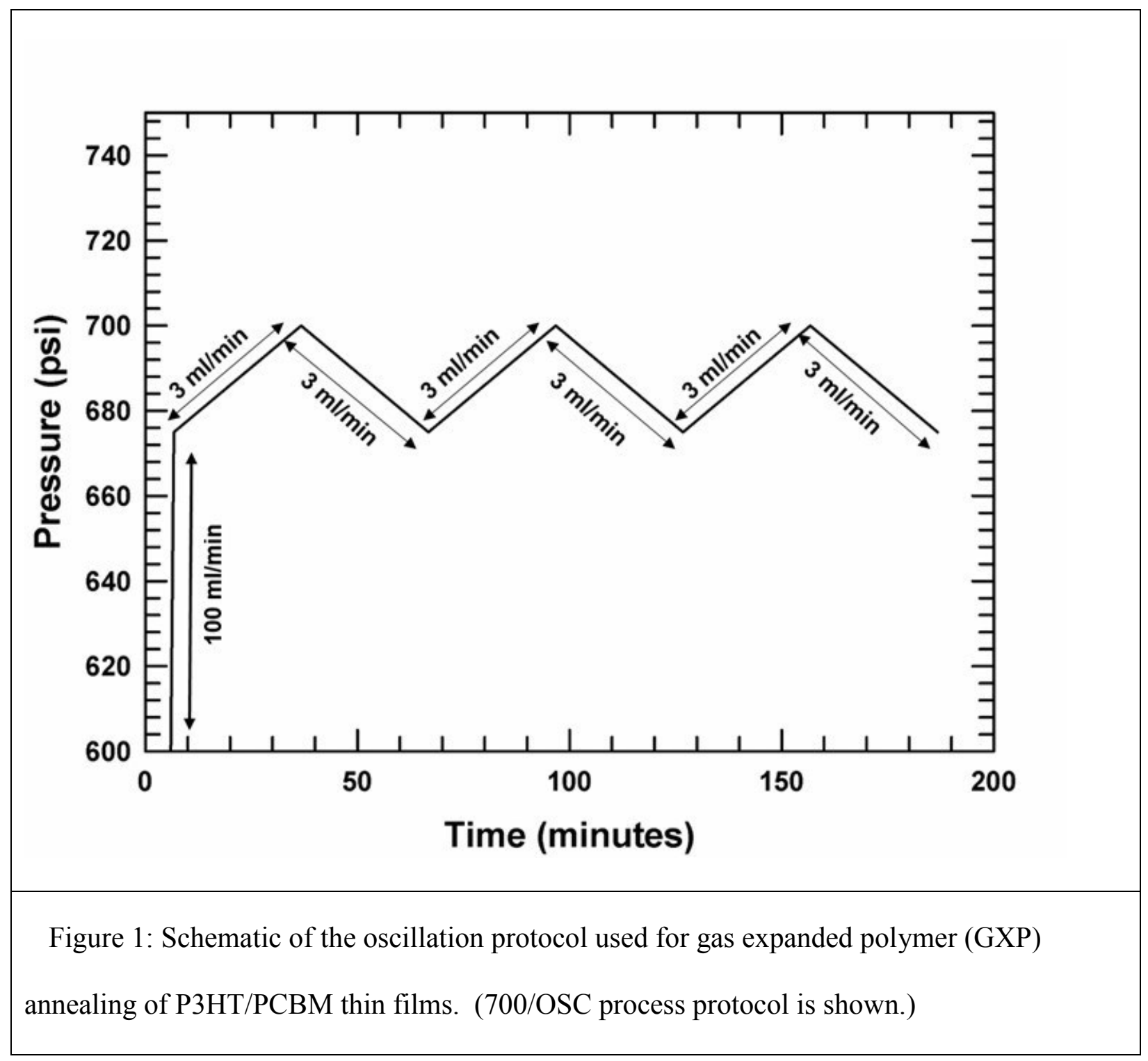




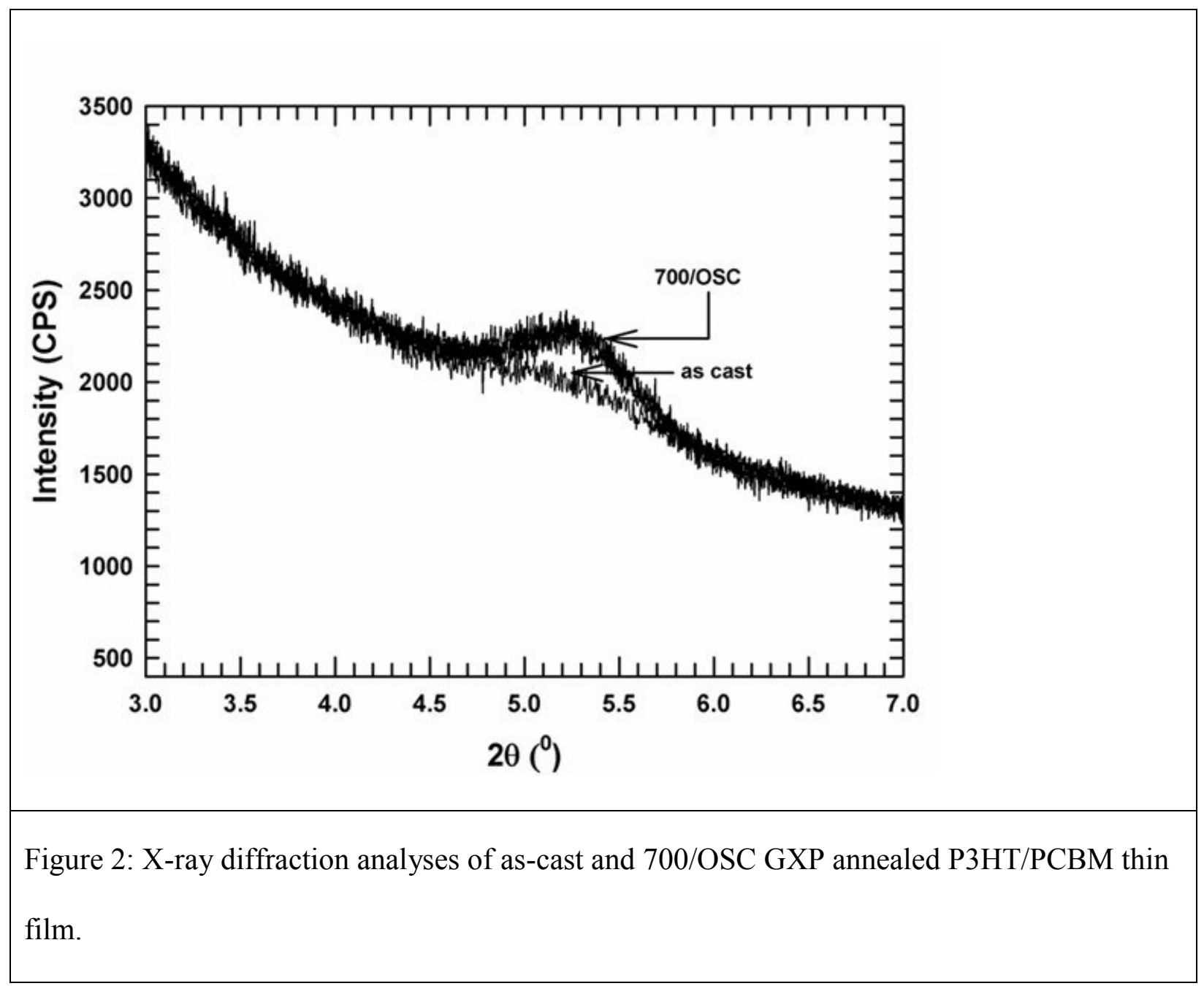




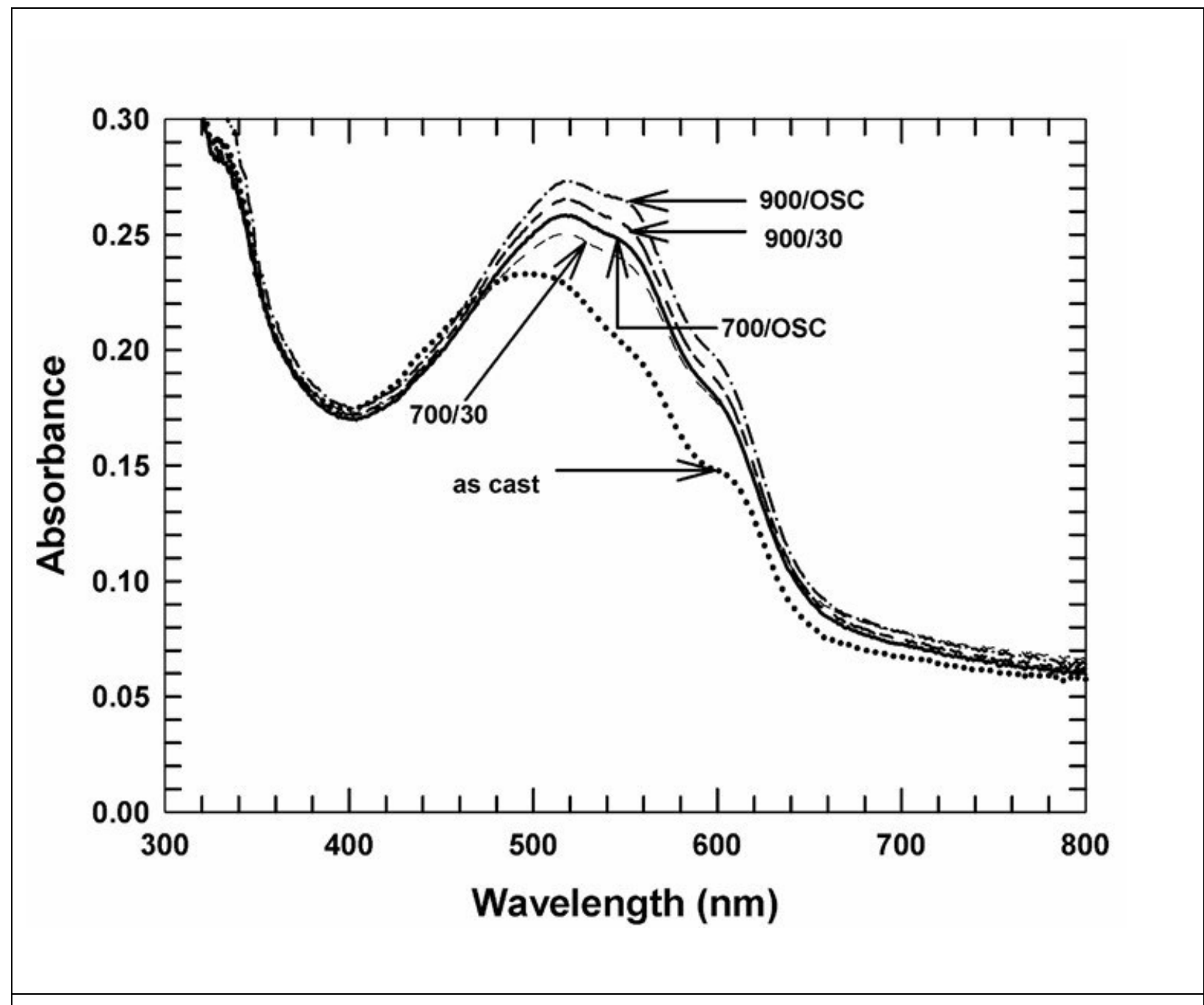

Figure 3: UV-Vis absorption spectra for as-cast and GXP annealed P3HT/PCBM thin films. 


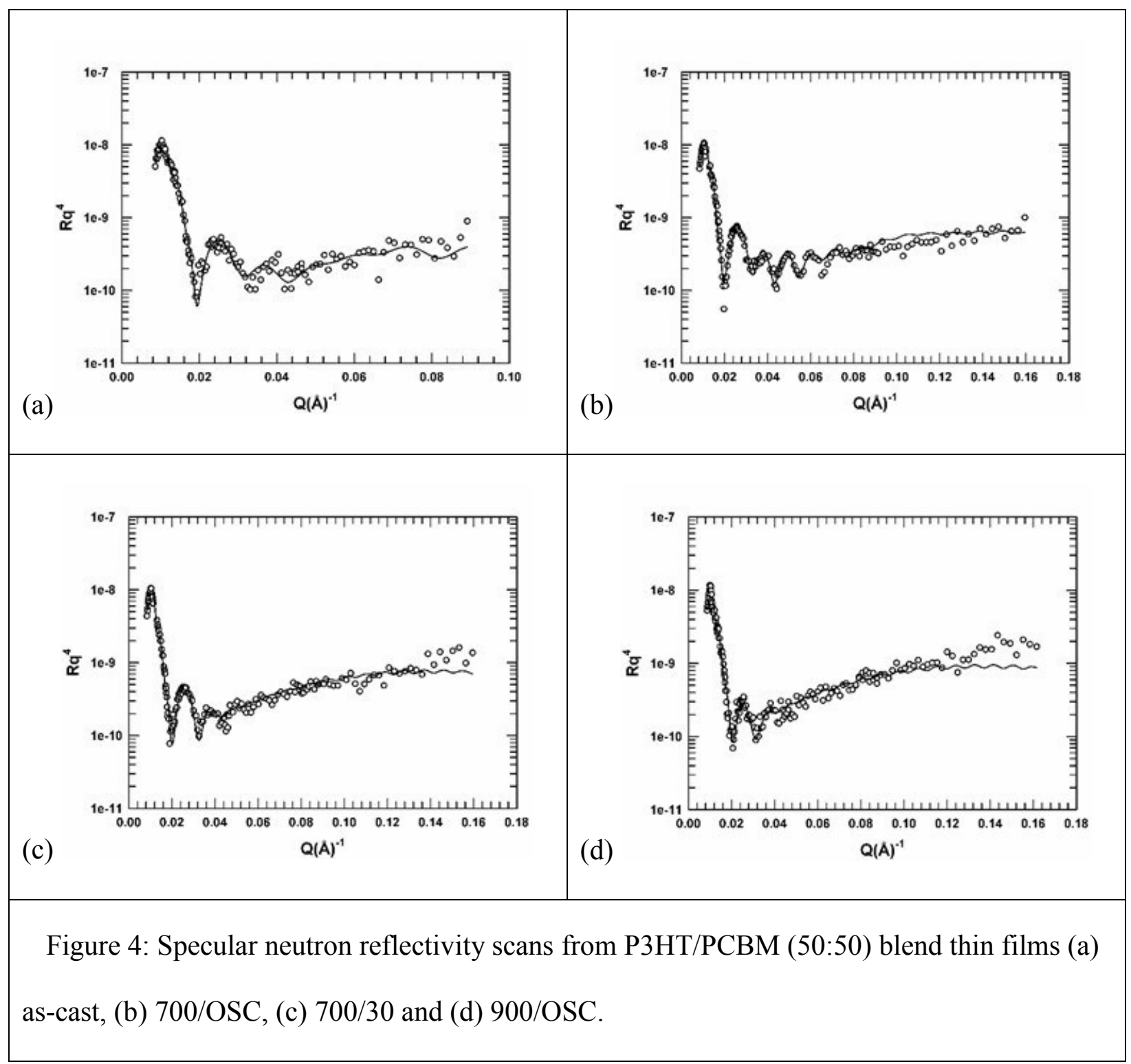




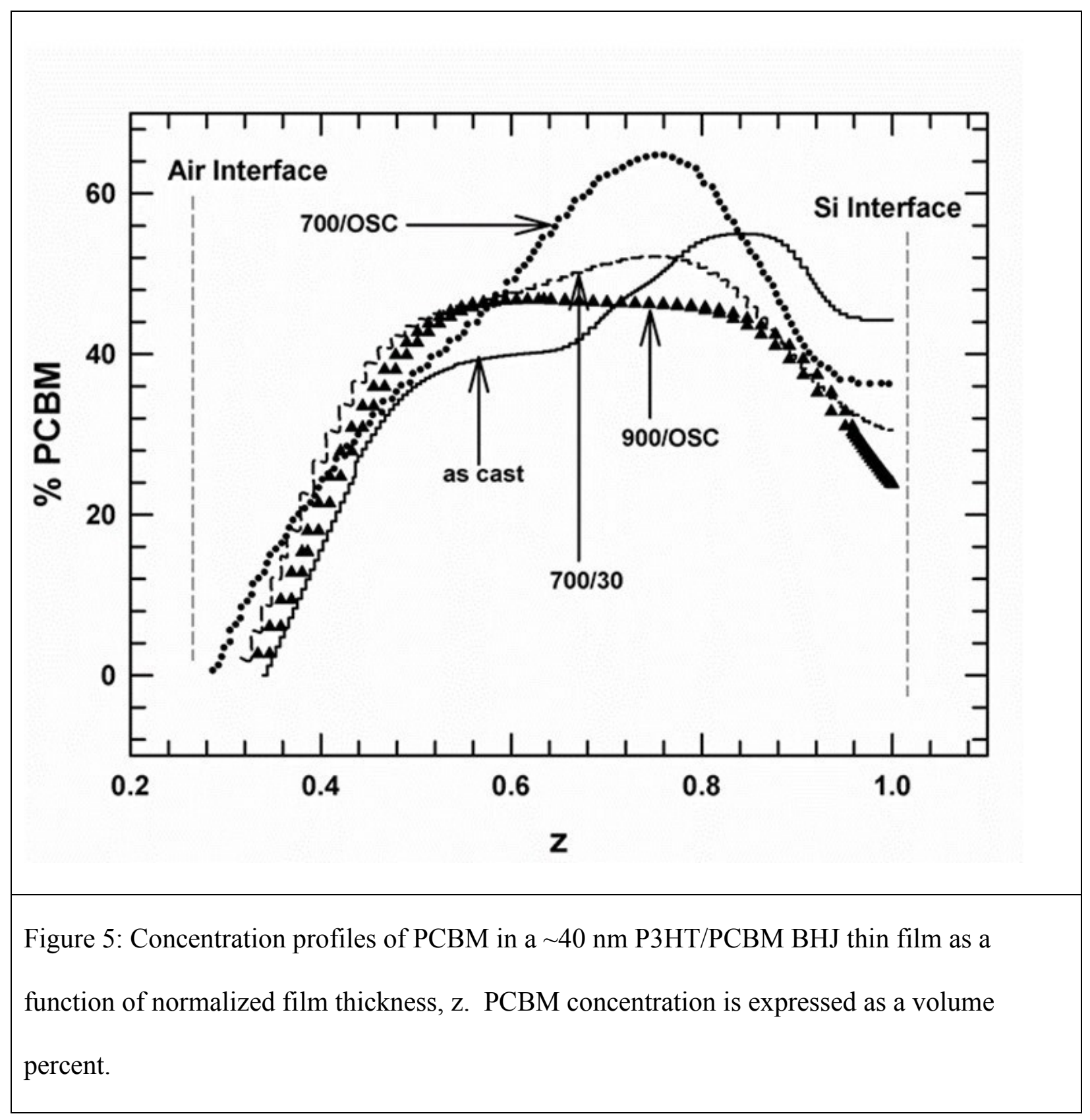


Table 1: Material Characterization

$$
\begin{array}{ccc}
\text { GXP Annealing Type } & \text { Contact } & \text { Thickness }(\AA)^{\mathrm{b}} \\
& \text { Angle }\left({ }^{0}\right)^{\mathrm{a}} \\
& +2
\end{array}
$$

\section{PCBM}

Oscillate pressure 675-700 psi 3 times

Steady pressure $700 \mathrm{psi}, 30 \mathrm{~min}$.

Oscillate pressure $875-900$ psi 3 times

Steady pressure $900 \mathrm{psi}, 30 \mathrm{~min}$.

Unannealed

P3HT
700/OSC

$700 / 30$

900/OSC

$900 / 30$

as cast
95.8

99

420

552

564

544

99.5

${ }^{\mathrm{a}}$ Contact angle measurements performed on films cast on glass.

${ }^{\mathrm{b}}$ Thicknesses were determined from fitting the neutron reflectivity data . 
Graphical Abstract

Gas Expanded Polymer Process to Anneal Nanoparticle Dispersion in Thin Films

Ambuken et al.
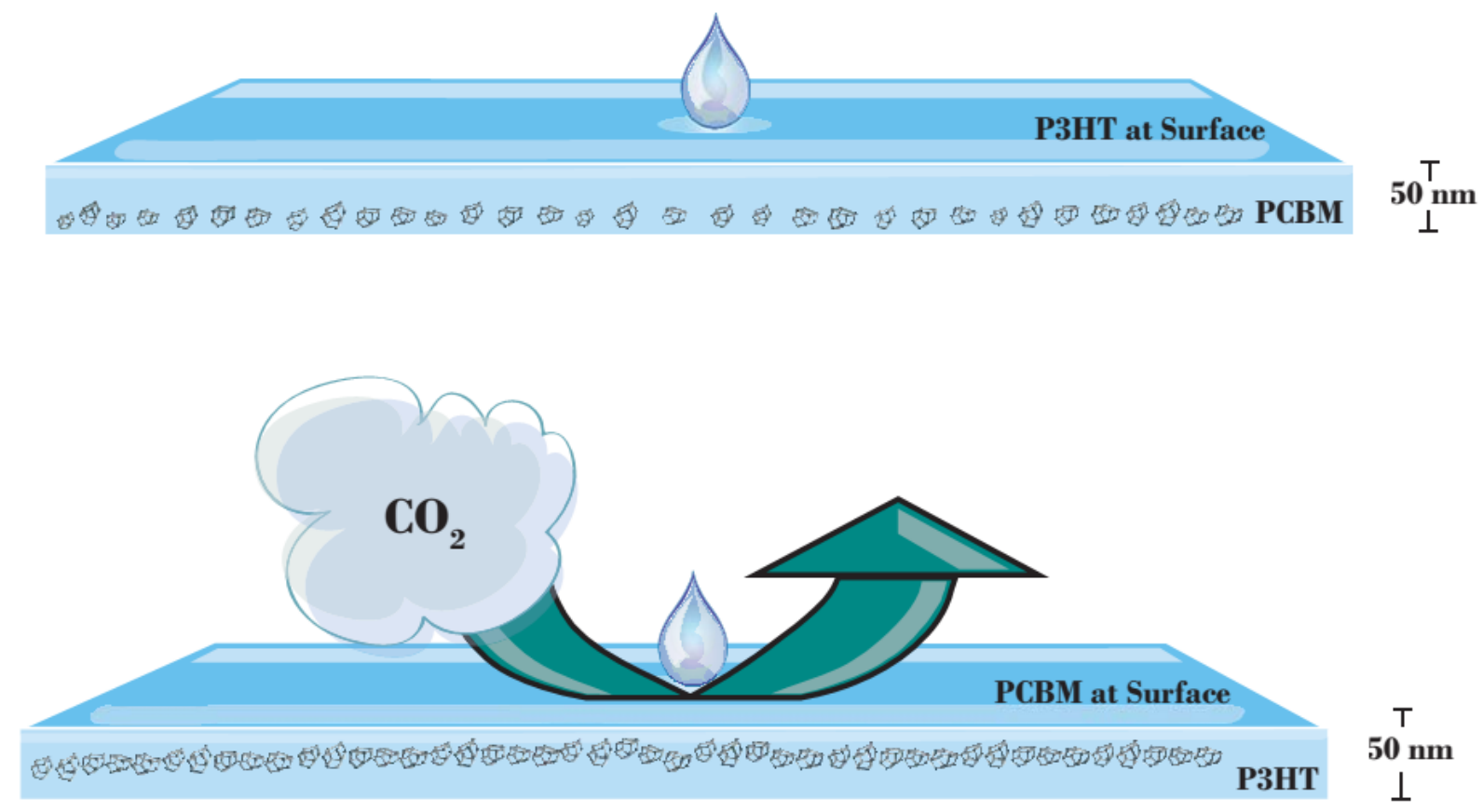